\title{
Surgical Treatment of Anterior Urethral Stricture Diseases: Brief Overview
}

\author{
Guido Barbagli, Massimo Lazzeri
}

Center for Reconstructive Urethral Surgery, Arezzo and Department of Urology, Santa Chiara Firenze, Florence, Italy

\begin{abstract}
We performed an up-to-date review of the surgical techniques suggested for the treatment of anterior urethral strictures. References for this review were identified by searching PubMed and MEDLINE using the search terms "urethral stricture" or "urethroplasty" from 1995 to 2006. Descriptive statistics of the articles were provided. Meta-analyses or other multivariate designs were not employed. Out of 327 articles, 50 (15\%) were determined to be germane to this review. Eight abstracts were referenced as the authors of this review attended the meetings where the abstract results were presented, thus it was possible to collect additional information on such abstracts. Urethrotomy continues to be the most commonly used technique, but it does have a high failure rate and many patients progress to surgical repair. Buccal mucosa has become the most popular substitute material in urethroplasty; however, the skin appears to have a longer follow-up. Free grafts have been making a comeback, with fewer surgeons using genital flaps. Short bulbar strictures are amenable using primary anastomosis, with a high success rate. Longer strictures are repaired using ventral or dorsal graft urethroplasty, with the same success rate. New tools such as fibrin glue or engineered material will become a standard in future treatment. In reconstructive urethral surgery, the superiority of one approach over another is not yet clearly defined. The surgeon must be competent in the use of various techniques to deal with any condition of the urethra presented at the time of surgery.
\end{abstract}

Key words: urethral stricture; surgical procedures, operative; graft; tissue engineering

Int Braz J Urol. 2007; 33: 461-9

\section{INTRODUCTION}

Urethral strictures are a frequent source of lower urinary tract disorders in adults, such as urinary tract infection, acute urinary retention, high-pressure voiding leading to secondary bladder thickening and irritability and even bladder diverticula or perineal fistulas and abscess (1). Blunt perineal trauma, urethral catheterization or instrumentation, lichen sclerosus and sexually transmitted diseases are the most frequent causes of strictures (1). However, most causes of urethral strictures remain unknown, but they are probably the result of a remote unrecognized perineal trauma experienced during childhood(1).

Surgical treatment of urethral stricture diseases is a continually evolving process, and currently there is renewed controversy over the best means of reconstructing the urethra. Moreover, the superiority of one technique over another has not yet been clearly defined. Urologists must be familiar with the use of numerous and various surgical techniques to deal with any condition of the urethra during surgery. This re- 
view aims to provide an update on the reconstructive techniques currently used to repair anterior urethral strictures and offer some insight on possible future strategies.

\section{MATERIALS AND METHODS}

Inclusion criteria for the literature review were operationalized before the initial literature search. The search incorporated original and review articles presenting data regarding all aspects of the surgical repair of anterior urethral strictures. The exclusion criteria included articles presenting opinions rather then evidence and articles that were not published in peer reviewed journals. The articles reviewed were limited to English language publications. A review of the world literature was performed via the MEDLINE/ PubMed databases using the search terms "urethral stricture" or "urethroplasty" from January 1995 through August 2006. The bibliographies of all relevant articles were reviewed for applicable citations that might not have appeared upon the database search. The authors independently reviewed each abstract identified by the database searches and relevance to the topic was ascertained.

\section{RESULTS}

A total of 327 articles were identified in our search and 50 of these (15\%) were determined to be germane to the topic under review. The bibliographies of all 50 articles were scanned and references that were not hits in our initial database search were also reviewed. Four articles were included from the bibliography search in the literature review. Eight abstracts were referenced as the authors of this review were in attendance at the meetings where the abstract results were presented, thus it was possible to collect additional information on the presented abstracts. A meta-analysis or other multivariate designs could not be correctly employed due to the heterogeneous nature of the data in the articles reviewed. Reasons for this are that standard diagnosis, success and complication criteria vary among authors. Therefore, only descriptive statistics of the articles are provided in this review.

\section{TREATMENT OF URETHRAL STRICTURES}

Peterson and Webster suggested that treatment for urethral strictures include numerous options, such as dilation, urethrotomy, stent and reconstructive surgical techniques and emphasized that no one technique is appropriate for all stricture diseases (2).

\section{Internal Urethrotomy vs. Open Urethroplasty}

Dilation and urethrotomy continue to be the most commonly used techniques, but they have a high failure rate with recurrence in $47.6 \%$ of patients and many patients progress to surgical repair $(2,3)$. Moreover, repeated dilation or urethrotomy exacerbates scar formation, thus adding to stricture length and predisposing to a more difficult definitive open repair and a lower success rate (1-3). Persistent use of dilation or urethrotomy for the treatment of urethral strictures may be the result of unfamiliarity with literature and inexperience with urethroplasty surgery (3). Surprisingly, a recent survey of stricture management involving 424 urologists from the USA showed that only $21 \%$ to $29 \%$ indicated that they would refer a patient with a recurrent urethral stricture to another urologist for urethroplasty, while $34 \%$ elected to continue endoscopic management despite predictable failure. Seventy-four percent of urologists believed that literature supports the use of urethroplasty only after repeated endoscopic failure (4). Greenwell et al. recently developed an algorithm for the management of urethral strictures based on cost-effectiveness and concluded that repeat urethrotomy or dilation are neither clinically effective nor cost-effective and can no longer be justified (3). Wright et al. determined, using decision analysis, the cost-effectiveness of different management strategies for short bulbar urethral strictures and concluded that the most cost-effective strategy for the management of short bulbar urethral stricture is to reserve urethroplasty for patients in whom a single endoscopic attempt has failed (5). Instead, for strictures for which the success rate of an urethrotomy is expected to be less than 35\%, applying urethroplasty as the primary therapy is costeffective (5). 
Open urethroplasty is regarded as the gold standard treatment for urethral strictures $(1,2)$. Nevertheless, urethroplasty is not a routine operation and a lack of the necessary skills should prompt a referral to a specialist skilled in urethroplasty (3). Unfortunately, most urologists have little experience with urethroplasty surgery and erroneously believe that the use of these complex reconstructive urethral procedures are justified only in young, healthy patients, since these treatments are associated to a high rate of complications requiring longer patient hospitalization and higher hospital costs. Internal urethrotomy is a lessinvasive outpatient procedure, providing the obvious benefits of surgeon/patient convenience and cost control. Recent literature, however, shows that urethroplasty can also be considered a "minimally" invasive technique and a more efficient therapy than internal urethrotomy. Santucci et al. reviewed 70 open urethroplasties performed on males older than 64 years old and concluded that urethroplasty should not be withheld solely on the basis of age, as older men tolerate urethroplasty well and complication rates are low (6). MacDonald et al. presented the review of 54 patients who underwent anterior urethroplasty to evaluate the safety and feasibility of decreasing the impact of urethroplasty by minimizing operative time, maximizing adjuvant pain therapy and using anesthetic agents that decrease the incidence and severity of side effects (7). The authors showed that urethroplasty could be safely performed with less than 24hours hospital stay and concluded that anterior urethroplasty performed as a same-day procedure appeared to be safe and well tolerated, without compromising functional outcome, and it costs $40 \%$ to $60 \%$ less than the same procedure performed on in-patients. Finally, Rourke \& Jordan suggested that treatment for a $2 \mathrm{~cm}$ bulbar urethral stricture with primary open urethroplasty is less costly than endoscopic treatment with internal urethrotomy (8).

\section{Urethral Tissue Transferred Material: Penile Skin vs. Buccal Mucosa}

Buccal mucosa has become the most popular substitute material in the treatment of urethral strictures, as it is readily available and easily harvested from the cheek or lip, allowing for a concealed donor site scar and low oral morbidity (9). Buccal mucosa is hairless, has a thick elastin-rich epithelium, which makes it tough yet easy to handle and a thin and highly vascular lamina propria, which facilitates inosculation and imbibition (9). Moreover, the use of buccal mucosa avoids cosmetic disadvantages and consequences caused by the use of genital skin. Prior to the use of buccal mucosa, penile skin was the preferred tissue transferred material used for urethroplasty. The question remains: is buccal mucosa really superior to penile skin? Alsikafi et al. in an effort to answer whether buccal mucosa is really the best, compared the outcome of 95 buccal mucosa urethroplasty and 24 penile skin graft urethroplasties (10). The overall success rate of penile skin urethroplasty was $84 \%$ (mean follow-up 201 months), while the success rate of buccal urethroplasty was $87 \%$ (mean follow-up 48 months) and no statistically significant difference was found between the two groups (10). Gozzi et al. retrospectively evaluated the results on 194 patients with glanular (20.6\%), penile (16\%), bulbar (20.1\%), membranous (29.4\%) and post-hypospadias repair (13.9\%) urethral strictures (11). All patients were treated by dorsal onlay techniques with genital and extra-genital skin grafts and reported excellent results with a $2 \%$ restenosis rate and a mean followup of 31 months (11). We retrospectively reviewed the outcome of 95 patients who underwent bulbar substitution urethroplasty, 45 receiving penile skin grafts (12) and 50 buccal mucosa grafts (13). Thirtythree of the 45 penile skin urethroplasties were successful $(73 \%)$ and $12(27 \%)$ were failures (12). Forty-two of the 50 buccal mucosal urethroplasty were successful (84\%) and $8(16 \%)$ were failures (13). The skin graft urethroplasty showed a higher failure rate $(27 \%)$ compared to the buccal mucosa graft $(16 \%)$, with the penile skin grafts having a longer follow-up (mean 71 months) compared to the buccal mucosa grafts (mean 42 months) $(12,13)$. In conclusion, skin and buccal mucosa are both excellent materials for urethroplasty with a comparable success rate, though the use of skin appears to have a longer follow-up than buccal mucosa.

\section{Penile Urethral Reconstruction}

Basically, the surgical technique for penile urethral reconstruction is selected according to the etiology of the urethral stricture disease and must also 
be based on the proper anatomic characteristics of the penile tissues to ensure flap or graft take and survival (14). Furthermore, sexual function can be placed at risk by any surgery on the genitalia and dissection must avoid interference with the neurovascular supply to the penis. The use of flaps or grafts, in single or multi-stage repair, should not compromise penile length or cause penile chordee, and certainly should not untowardly affect penile appearance. Penile urethroplasty could be a simple procedure in patients with a normal penis, but it can be a difficult challenge in men with strictures associated to genital lichen sclerosus or following failed hypospadias repair. Regardless, penile urethroplasty, be it a single or multi-stage repair, is intrinsically prone to complications such as edema, hematoma or infection, which in turn can lead to secondary complications, such as fistula or tissue necrosis, and it is the procedure most likely to produce alterations in sexual functions.

\section{Flaps vs. Grafts}

The controversy over the best means of reconstructing the penile urethra has been renewed and in recent years, free grafts have been making a comeback, with fewer surgeons using genital flaps (15-17). Rarely, has the current literature provided us with prospective studies comparing the grafts with the flaps, making it hard to declare a clear favorite (16). At present, we are uncertain in which patients the use of a pedicled flap will have better chances of success than a free graft, as the thin penile corpus spongiosum and the dartos fascia do not ensure sufficient graft support in all patients $(15,16)$. Identification and use of criteria to more carefully select the appropriate procedure for the patient might clarify whether the use of a graft is preferable to the use of a flap according to the characteristics of the vascular and mechanical tissues used to support the original urethral plate. Different authors recently described a new one-stage penile urethroplasty that involves a deeply longitudinal midline incision of the urethral plate and the suturing of buccal mucosal tissue as an inlay graft into the bed obtained within the urethral plate $(15,18,19)$. Unfortunately, the long-term results in a large series of patients treated with this new one-stage penile graft urethroplasty are, at the moment, not available in the current literature.

\section{One-Stage vs. Two-Stage Repair}

Penile urethroplasty should be performed in a single-stage whenever possible to avoid patient discomfort and disability that can be caused by the use of multi-stage procedures. In patients with urethral strictures caused by trauma, infection, instrumentation or catheter, the penis is generally normal and the penile skin, urethral plate, corpus spongiosum and dartos fascia are suitable for urethral reconstruction. In such cases, one-stage urethroplasty is the surgery of choice. Instead, in patients who have experienced failed hypospadias repair or in whom the penile skin, urethral plate and dartos fascia are not suitable for urethral reconstruction, two-staged urethroplasty is recommended $(20,21)$. In addition, in patients suffering from genital lichen sclerosus, the use of buccal mucosa is mandatory since, as a skin disease, any skin that would be used as graft material is already or may become diseased $(22,23)$. When used in a multistage procedure, the buccal mucosa or skin grafts do not heal in the same way in all patients and numerous revisions of the graft-bed may be necessary to obtain a satisfactory mucosal bed before the urethral closure (21). Unfortunately, these repeated surgical revisions of the scars could have a tremendous psychological impact on the patient (21).

\section{Bulbar Urethral Reconstruction}

Basically, the surgical technique used in the repair of the bulbar urethral stricture is selected according to stricture length (14). Strictures ranging from 1 to $2 \mathrm{~cm}$ are treated by using end-to-end anastomosis; strictures ranging from 2 to $3 \mathrm{~cm}$ are managed using augmented roof-strip anastomosis; strictures longer than $3 \mathrm{~cm}$ are treated using substitution urethroplasty. Finally, in patients with strictures associated to local adverse conditions (fistula, abscess, tumor, stent, or previous failed urethroplasty) multi-stage urethroplasty is mandatory.

\section{End-to-End Anastomosis}

Short strictures in the bulbar urethra are generally amenable to complete excision with primary anastomosis via a perineal incision, affording a high success rate of 95\%, as reported by Santucci et al. (24). The surgical technique of end-to-end anastomosis was recently illustrated step by step by Mundy 
with the use of nicely executed color drawings and excellent commentary (25). Recently, the stricture length ideal for the application of end-to-end anastomosis has become a contentious issue. Guralnick \& Webster suggested that end-to-end anastomosis is appropriate only for a bulbar stricture of $1 \mathrm{~cm}$ or less as excision of a $1 \mathrm{~cm}$ urethral segment with opposing $1 \mathrm{~cm}$ proximal and distal spatulation results in a $2 \mathrm{~cm}$ urethral shortening, which may be adequately accommodated by the elasticity of the mobilized bulbar urethra without chordee (26). The authors emphasized that excision of a longer urethral segment risks penile shortening or chordee, even if lengthening maneuvers are applied (26). On the contrary, Morey \& Kizer reported 22 patients with proximal bulbar urethral strictures greater that $2.5 \mathrm{~cm}$ long (range 2.6 to $5 \mathrm{~cm}$ ) that were managed using an extended anastomotic approach, suggesting that the possibility of reconstructing the urethra is proportional to the length and elasticity of the distal urethral segment (27). They reported a $91 \%$ success rate with a mean follow-up of 22.1 months, and with no increase in erectile complaints compared to shorter strictures (27). Finally, Al-Qudah $\&$ Santucci suggested that the use of end-to-end anastomosis is also controversial in the treatment of short and medium length urethral strictures (range 0.5 to $3.0 \mathrm{~cm})(28)$. They presented 47 short urethral strictures treated with end-to-end anastomosis or buccal mucosal onlay graft urethroplasty and compared early and intermediate outcomes to determine which was the best technique (28). The recurrence rate was 7\% in those patients who underwent end-to-end anastomosis and $0 \%$ in patients who underwent buccal mucosal graft urethroplasty. Early and late major complications occurred in $18 \%$ of the patients after anastomotic repair, including penile chordee and erectile dysfunction (28). In conclusion, buccal mucosal onlay graft urethroplasty is suggested as the operation of choice even for short urethral strictures (28).

\section{Augmented Roof Strip Anastomosis}

In 1998, Iselin and Webster modified our original technique of dorsal onlay urethroplasty (29). In this procedure, the worst section of the stricture is removed and the urethra is re-anastomosed and dorsally augmented with a free graft (29). The surgical technique of augmented roof-strip anastomosis was recently illustrated step by step by Mundy, who also included an excellent commentary (29). In 2004, Delvecchio et al. suggested that the use of augmented roof-strip anastomotic urethroplasty incorporating the graft onlay into the receiving urethral plate is less successful, either because of the inherent deterioration of transferred tissues exposed to urine or to the fact that the onlay is performed in an area of dense spongiofibrosis, generally at the site the stricture disease originated, which is unsuitable for simple onlay grafting (30). These authors proposed always excising this area, followed by direct reanastomosis of the floor strip and onlay of the adjacent "better" stricture, whatever its length (30). The authors showed that this technique had only a 5.2\% failure rate in 38 patients, compared with a $9 \%$ failure rate in 11 patients who underwent a simple augmented graft urethroplasty without excision of the strictured tract. They concluded that excision of the worst stricture segment avoids a long onlay in a poor urethral bed where failure often occurs at the location of even the smallest stricture caliber (30). Augmented roof strip anastomotic repair may be arranged using ventral or dorsal graft location. In 2005, Abouassaly \& Angermeier reported the results of 36 patients undergoing augmented anastomotic repair with ventral onlay grafts and 4 patients undergoing augmented anastomotic repair with dorsal onlay grafts and concluded that ventral or dorsal onlay seems to offer comparable results (31). In 2006, Abouassaly and Angermeier recommended complete excision of the stricture and use of an augmented roofstrip anastomotic repair for strictures that cover a particularly narrow area of $1-2 \mathrm{~cm}$ in length (32). Out of 69 patients, 63 were successful $(91 \%)$ with a mean follow-up of 34 months (32).

\section{Substitution Urethroplasty Using Buccal Mucosal Graft}

Buccal mucosal urethroplasty represents the most widespread method for the repair of strictures in the bulbar urethra, due to its highly vascular tissue. Location of the graft on the urethra surface has become a contentious issue (33), dating from the time we described dorsal onlay graft urethroplasty techniques (34). Wessells \& Armenakas suggested a list of the technical advantages of ventral onlay urethroplasty: complete circumferential mobilization of the 
urethra is not necessary, thus preserving arterial and venous connections to the corpora cavernosa; stricture is easily seen; performance of a urethrotomy allows the lumen to be clearly delineated, thus allowing the surgeon to identify mucosal edges, measure the size of the plate, carry out a watertight anastomosis and, if necessary, excise portion of the stricture and perform dorsal re-anastomosis $(35,36)$.

Success with bulbar buccal mucosal grafts has been high with dorsal $(13,33)$ or ventral graft location $(35,36)$ and the different graft positions have shown no difference in success rate $(13,31)$. Recently, Abouassaly and Angermeier reported the intermediate term results on 100 patients with penile (21\%), bulbar (82\%) and bulbomembranous (17\%) urethral strictures undergoing anterior and posterior buccal mucosal graft urethroplasty using different graft locations (ventral or dorsal) (31). These patients had a final success rate of $92 \%$ (mean followup 29.5 months) (31). In our experience, the placement of the grafts on the ventral, dorsal or lateral surface of the bulbar urethra provided the same success rates (83\% to $85 \%)$ and stricture recurrence was uniformly distributed in all patients (13). Recently, we reviewed the patterns of failure following bulbar substitution urethroplasty and investigated the prevalence and location of anastomotic fibrous ring strictures occurring at the apical anastomosis between the graft and urethral plate (37). Out of 107 patients, $85(80 \%)$ were successful and $22(20 \%)$ failured. Failure in 12 patients $(11 \%)$ involved the entire grafted area and in 10 patients (9\%) it involved the anastomotic site (5 distal, 5 proximal). The prevalence and location of these anastomotic ring strictures were uniformly distributed among the three different surgical techniques, using either skin or buccal mucosal grafts (37). Others authors found these anastomotic fibrous ring strictures after substitution onlay urethroplasty (37).

\section{Use of Fibrin Glue in Urethral Reconstruction}

Fibrin glue contains fibrinogen, Factor XII, plasmafibronectina and plasminogen dissolved in an aprotin solution (bovine) with an activate thrombin component (human) mixed with a calcium chloride solution. When combined, a dense gelatinous clot is quickly formed at the point of application. Because this fibrin sealant is non-synthetic and, therefore biocompatible with the natural fibrinolytic mechanism, healing is promoted without inflammation and fibrosis formation (38). Several studies emphasized the use of fibrin glue in tissue-engineered procedures $(39,40)$. The use of fibrin sealant is widely published in the literature. Since this sealant is composed of human products, the plasma is screened, tested and thermally treated to ensure viral safety (41).

The application of fibrin glue in surgery mainly relates to its sealing power. It has been shown to be a beneficial adjunct to sutures for closing wounds and promoting healing since it increases tissue plane adherence, accelerates revascularization, reduces hemorrhage, prevents seroma formation and decreases inflammation. The published urological literature has recently contained an increasing number of studies suggesting the use of fibrin glue in reconstructive genital and urethral procedures. In 2002, DeCastro \& Morey described the use of fibrin tissue adhesive in genital skin loss due to Fournier's gangrene (42). In 2003, Evans et al. reported the use of fibrin sealant to manage iatrogenic urinary tract injuries, urinary fistulas and surgical complications (38). In 2004, Hick and Morey assessed whether fibrin sealant promotes early catheter removal after urethral reconstruction (43). In 2006, Morris et al. reported the use of fibrin glue in the reconstruction of genital skin loss (44). We recently reported our experience with the use of fibrin glue in bulbar urethral reconstruction in a series of patients who underwent augmented anastomotic repair (45) or dorsal onlay graft urethroplasty (46). However, further comparative studies are necessary to confirm that the use of fibrin glue is really beneficial and to evaluate whether its use reduces restenosis rate following substitution urethroplasty $(45,46)$.

\section{Tissue Engineering Urethroplasty}

McAninch recently emphasized that urethral reconstruction can require some of the most challenging techniques in urological surgery and excellent results can be obtained with today's techniques, but it would be a significant advantage to have tissue-engineered products for urethroplasty (47). Carson suggested that urethroplasty represents a model of international progress in urology and the field of urethral stricture 
repair has matured greatly with a growing number of single-stage repairs being performed with continued improvements in patient outcome (48). Moreover, the use of tissue engineering to optimize graft material may allow us to combine the most refined surgical techniques with the best graft material, to archive even more reliable results (48).

Ribero-Filho et al. recently presented a new urethroplasty technique that uses human cadaveric urethral acellular matrix (49). After having been harvested from a cadaveric donor the urethral mucosa and spongiosum tissue were enzymatically converted into a urethral acellular matrix graft (49). The graft was applied onto the urethra as a ventral onlay patch. No immunosuppressors were necessary, there were no postoperative complications and the final outcome was satisfactory (49). Could it be that we have reached the limit of this veteran workhorse of substitution urethroplasty? (50). The time has arrived to look beyond buccal mucosa to the development of other forms of substitution material, incorporating tissue engineered materials or stem cells into our quest for the Holy Grail of urethral substitution (50).

\section{CONCLUSION}

Reconstructive urethral surgery must better adapt to the characteristics of the disease if the features defining its professionalism are to be strengthened: control over setting standards, improvement of minimally invasive procedures, research and translation of the basic scientific results into daily clinical practice, and imposing the responsibility for organizing, appraising and maintaining quality patient care.

\section{CONFLICT OF INTEREST}

None declared.

\section{REFERENCES}

1. Waxman SW, Morey AF: Management of urethral strictures. Lancet. 2006; 367: 1379-80.
2. Peterson AC, Webster GD: Management of urethral stricture disease: developing options for surgical intervention. BJU Int. 2004; 94: 971-6.

3. Greenwell TJ, Castle C, Andrich DE, MacDonald JT, Nicol DL, Mundy AR: Repeat urethrotomy and dilation for the treatment of urethral stricture are neither clinically effective nor cost-effective. J Urol. 2004; 172: 275-7.

4. Brandes SB, Smith J, Virgo K, Johnson FE: Adult anterior urethral strictures: a national practice patterns survey. J Urol. 2001; 165: 13.

5. Wright JL, Wessells H, Nathens AB, Hollingworth W: What is the most cost-effective treatment for 1 to $2-\mathrm{cm}$ bulbar urethral strictures: societal approach using decision analysis. Urology. 2006; 67: 889-93.

6. Santucci RA, McAninch JW, Mario LA, Rajpurkar A, Chopra AK, Miller KS, et al.: Urethroplasty in patients older than 65 years: indications, results, outcomes and suggested treatment modifications. J Urol. 2004; 172: 201-3.

7. MacDonald MF, Al-Qudah HS, Santucci RA: Minimal impact urethroplasty allows same-day surgery in most patients. Urology. 2005; 66: 850-3.

8. Rourke KF, Jordan GH: Primary urethral reconstruction: the cost minimized approach to the bulbous urethral stricture. J Urol. 2005; 173: 1206-10.

9. Barbagli G, Palminteri E, De Stefani S, Lazzeri M: Harvesting buccal mucosal grafts. Keys to success. Contemp Urol. 2006; 18: 17-24.

10. Alsikafi NF, Eisenberg M, McAninch JW: Long-term outcomes of penile skin graft versus buccal mucosal graft for substitution urethroplasty of the anterior urethra. J Urol. 2005; 173:87.

11. Gozzi C, Pelzer AE, Bartsch G, Rehder P: Genital free skin grafts as dorsal onlay for urethral reconstruction. J Urol. 2006; 175: 38.

12. Barbagli G, Palminteri E, Lazzeri M, Turini D: Interim outcomes of dorsal skin graft bulbar urethroplasty. J Urol. 2004; 172: 1365-7.

13. Barbagli G, Palminteri E, Guazzoni G, Montorsi F, Turini D, Lazzeri M: Bulbar urethroplasty using buccal mucosa grafts placed on the ventral, dorsal or lateral surface of the urethra: are results affected by the surgical technique? J Urol. 2005; 174: 955-7.

14. Barbagli G, Palminteri E, Lazzeri M, Guazzoni G: Anterior urethral strictures. BJU Int. 2003; 92: 497-505.

15. Barbagli G, Palminteri E, De Stefani S, Lazzeri M: Penile urethroplasty. Techniques and outcomes using buccal mucosa grafts. Contemp Urol. 2006; 18: 2533. 
16. Wessells H, McAninch JW: Current controversies in anterior urethral stricture repair: free-graft versus pedicled skin-flap reconstruction. World J Urol. 1998; 16: $175-80$.

17. Wessells H, McAninch JW: Use of free grafts in urethral stricture reconstruction. J Urol. 1996; 155: 1912-5.

18. Asopa HS, Garg M, Singhal GG, Singh L, Asopa J, Nischal A: Dorsal free graft urethroplasty for urethral stricture by ventral sagittal urethrotomy approach. Urology. 2001; 58: 657-9.

19. Gupta NP, Ansari MS, Dogra PN, Tandon S: Dorsal buccal mucosal graft urethroplasty by a ventral sagittal urethrotomy and minimal-access perineal approach for anterior urethral stricture. BJU Int. 2004; 93: 1287 90.

20. Bracka A: Hypospadias repair: the two-stage alternative. Br J Urol. 1995; 76 Suppl 3: 31-41.

21. Barbagli G, De Angelis M, Palminteri E, Lazzeri M: Failed hypospadias repair presenting in adults. Eur Urol. 2006; 49: 887-94.

22. Venn SN, Mundy AR: Urethroplasty for balanitis xerotica obliterans. Br J Urol. 1998; 81: 735-7.

23. Depasquale I, Park AJ, Bracka A: The treatment of balanitis xerotica obliterans. BJU Int. 2000; 86: 459-65.

24. Santucci RA, Mario LA, McAninch JW: Anastomotic urethroplasty for bulbar urethral stricture: analysis of 168 patients. J Urol. 2002; 167: 1715-9.

25. Mundy AR: Anastomotic urethroplasty. BJU Int. 2005; 96: 921-44.

26. Guralnick ML, Webster GD: The augmented anastomotic urethroplasty: indications and outcome in 29 patients. J Urol. 2001; 165: 1496-501.

27. Morey AF, Kizer WS: Proximal bulbar urethroplasty via extended anastomotic approach—what are the limits? J Urol. 2006; 175: 2145-9.

28. Al-Qudah HS, Santucci RA: Buccal mucosal onlay urethroplasty versus anastomotic urethroplasty (AU) for short urethral strictures: which is better? J Urol. 2006; 175: 103.

29. Iselin CE, Webster GD: Dorsal onlay urethroplasty for urethral stricture repair. World J Urol. 1998; 16: 181-5.

30. Delvecchio FC, Anger JT, Webster GD: A proposal that whenever possible stricture excision be a part of all bulbar urethroplasties: A progressive approach to patient selection. J Urol. 2004; 171: 17.

31. Abouassaly R, Angermeier KW: Cleveland clinic experience with buccal mucosa graft urethroplasty: intermediate-term results. J Urol. 2005; 173: 33.

32. Abouassaly R, Angermeier KW: Augmented anastomotic urethroplasty (AAR) in patients with dense urethral stricture disease. J Urol. 2006; 175: 38.
33. Andrich DE, Leach CJ, Mundy AR: The Barbagli procedure gives the best results for patch urethroplasty of the bulbar urethra. BJU Int. 2001; 88: 385-9.

34. Barbagli G, Selli C, Tosto A, Palminteri E: Dorsal free graft urethroplasty. J Urol. 1996; 155: 123-6.

35. Wessells H: Ventral onlay graft techniques for urethroplasty. Urol Clin North Am. 2002; 29: 381-7.

36. Armenakas NA: Long-term outcome of ventral buccal mucosal grafts for anterior urethral strictures. AUA News 2004; 9: 17-8.

37. Barbagli G, Guazzoni G, Palminteri E, Lazzeri M: Anastomotic fibrous ring as cause of stricture recurrence after bulbar onlay graft urethroplasty. J Urol. 2006; 176: 6149.

38. Evans LA, Ferguson KH, Foley JP, Rozanski TA, Morey AF: Fibrin sealant for the management of genitourinary injuries, fistulas and surgical complications. J Urol. 2003; 169: 1360-2.

39. Schoeller T, Neumeister MW, Huemer GM, Russell RC, Lille S, Otto-Schoeller A, et al.: Capsule induction technique in a rat model for bladder wall replacement: an overview. Biomaterials. 2004; 25: 1663-73.

40. Currie LJ, Sharpe JR, Martin R: The use of fibrin glue in skin grafts and tissue-engineered skin replacements: a review. Plast Reconstr Surg. 2001; 108: 1713-26.

41. Morey AF, McDonough RC 3rd, Kizer WS, Foley JP: Drain-free simple retropubic prostatectomy with fibrin sealant. J Urol. 2002; 168: 627-9.

42. DeCastro BJ, Morey AF: Fibrin sealant for the reconstruction of fournier's gangrene sequelae. J Urol. 2002; 167: 1774-6.

43. Hick EJ, Morey AF: Initial experience with fibrin sealant in pendulous urethral reconstruction. Is early catheter removal possible? J Urol. 2004; 171: 1547-9.

44. Morris MS, Morey AF, Stackhouse DA, Santucci RA: Fibrin sealant as tissue glue: preliminary experience in complex genital reconstructive surgery. Urology. 2006; 67: 688-91.

45. Barbagli G, De Stefani S, Sighinolfi MC, Pollastri CA, Annino F, Micali S, et al.: Experience with fibrin glue in bulbar urethral reconstruction using dorsal buccal mucosa graft. Urology. 2006; 67: 830-2.

46. Barbagli G, De Stefani S, Sighinolfi MC, Annino F, Micali S, Bianchi G: Bulbar urethroplasty with dorsal onlay buccal mucosal graft and fibrin glue. Eur Urol. 2006; 50:467-74.

47. McAninch JW: Urethral reconstruction: a continuing challenge. J Urol. 2005; 173: 7.

48. Carson CC: Editorial. Urethroplasty: a model for international progress in urology. Contemp Urol. 2006; 18: 11. 


\title{
Anterior Urethral Stricture
}

49. Ribeiro-Filho LA, Mitre AI, Sarkis AS, Guimaraes PE, Arap MA, Silva IA, et al.: Human organ-specific acellular matrix grafting for severe urethral stenosis. J Urol. 2006; 175: 161 .
50. Armenakas NA: Editorial comment. J Urol. 2006; 176: 619.

Received: January 2, 2007

Accepted: March 5, 2007

\author{
Correspondence address: \\ Dr. Massimo Lazzeri \\ Department of Urology, Santa Chiara-Firenze \\ Piazza Indipendenza, 11 \\ Florence, 50129, Italy \\ E-mail: lazzeri.m@tiscali.it
}

